\title{
Scénario multimédia
}

\section{Formalisation moléculaire pour l'écriture de scénarii de documents multimédias}

\author{
Alain Durand*,** \\ * Univ Lille Nord de France, F-59000 Lille \\ ** UVHC, LSC, F-59313 Valenciennes \\ alain.durand@univ-valenciennes.fr
}

RÉSUMÉ. Les documents multimédias actuels connaissent une forme de standardisation avec une structure très hiérarchisée. Ceci résulte notamment du besoin de maîtriser les coûts de production, de la linéarité de représentation des concepteurs, et de l'absence de formalisation d'écriture adaptée aux possibilités du multimédia. Cette absence de représentation performante et la "linéarité par partie» issue de l'héritage littéraire empêchent la production de documents d'auteurs utilisant toutes les possibilités créatives offertes par le multimédia. Cet article présente dans un premier temps les fonctions du scénario multimédia pour qu'il puisse être un réel modèle du document, l'approche de représentation des documents afin de s'affranchir de la sclérose des liens hypertextes. Dans un second temps, la scénarisation moléculaire, adaptée à la représentation des documents multimédias, est développée afin de répondre aux critères définis dans la première partie.

ABSTRACT. Most of multimedia documents have a hierarchical structure. This results in particular, from the need to control production costs, from the linear representation of designers, and from the lack of formal writing adapted to the multimedia possibilities. This lack of high-performance representation and the literary heritage (with the "linear part by end") inhibit the design of new creative documents. In a first step, this article presents the functions of the multimedia scenario to avoid the hyperlinks sclerosis. In a second step, the molecular script, adapted to the representation of multimedia documents, is described to meet the criteria set out in the first part.

MOTS-CLÉS: scénario, multimédia, conception multimédia.

KEYWORDS: multimedia design, script, multimedia.

DOI:10.3166/DN.12.2.47-80 @ 2009 Lavoisier, Paris

RSTI - DN - 12/2009. Conception, design des documents numériques, pages 47 à 80 


\section{Introduction}

Depuis ses prémices jusqu'à aujourd'hui, le multimédia a subi de nombreuses évolutions. $\mathrm{Si}$ ces mutations sont souvent les conséquences d'avancées technologiques, les contraintes économiques ont aussi contribué à faire évoluer la forme des documents ${ }^{1}$ produits ainsi que les méthodes de travail pour y parvenir.

Dans l'euphorie des années 1990 de nombreuses sociétés de production multimédia ont été créées. L'absence de réelles méthodes de travail, d'évaluation des risques a conduit bon nombre d'entre elles à la faillite (Viéville, 2003). Cette expérience a entraîné une rationalisation « restrictive » de la production. En effet, la plupart des documents multimédias se limitent aujourd'hui à la forme hiérarchique d'un site internet de recherche d'information. En reproduisant les mêmes structures de documents, en appliquant des règles ergonomiques spécifiques, les risques de dérapages financiers sont limités. Il y a donc une standardisation des formes de documents et des méthodes employées pour les produire (Cartier, 2003). Malgré une spécification de méthodes et d'outils nécessaires à la conception des documents multimédias définie par la méthodologie AUTHOR (Huart, 2000), (cf. figure 2) et la conception de sites web efficaces en termes de communication (Rojas, 2007 ; Pignier et Drouillat, 2004), le processus d'écriture est bien souvent négligé. Dans un tel contexte, la créativité ne se matérialise guère qu'à travers le graphisme et les interfaces utilisateurs, et ce, même si les documents consultés à distance peuvent aujourd'hui rejoindre la complexité des documents installés sur un poste de travail.

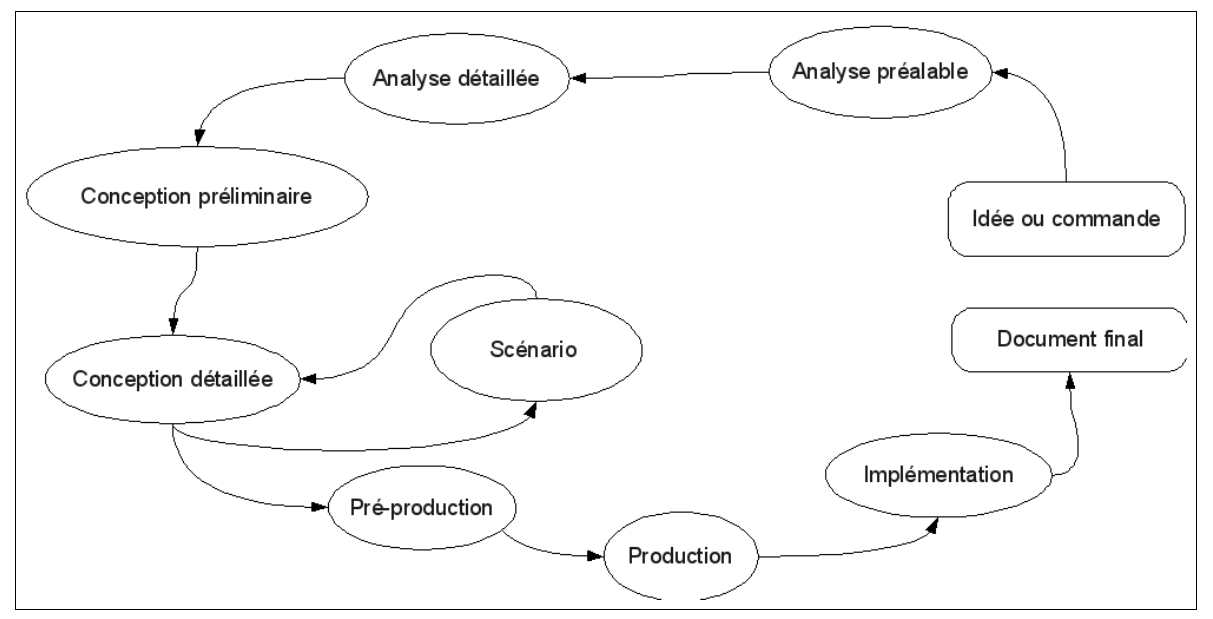

Figure 1. Ecriture du scénario au sein de la méthodologie AUTHOR

1. Un document est un produit de communication (Leleu-Merviel, 1997). 
L'objectif du travail présenté ici est de fournir un formalisme d'écriture, qui permette d'offrir des outils d'écriture. Pour reprendre les propos d'Yves Jeanneret et Emmanuel Souchier (1999), le modèle présenté vise à définir un outil d'écriture « d'architexte ».

Dans un premier temps, le scénario du document multimédia sera circonscrit puis, une fois les principes du modèle moléculaire définis, les modalités d'écriture et la façon dont l'auteur peut spécifier des interactions entre éléments du document seront décrites. L'objectif terminal est de fournir aux auteurs multimédias des outils de représentation de documents comportant des entités autonomes capables d'interagir entre elles et avec le lecteur.

\section{Le scénario}

Avant d'aborder la problématique du scénario au sein de la conception des documents multimédias, il est important de s'attacher à certaines des caractéristiques de la réalisation (fabrication) de ces documents.

\subsection{Quelques caractéristiques de la réalisation multimédia}

La réalisation multimédia nécessite la production de différents documents qui seront intégrés au document final avec des fonctions informatiques permettant une interaction entre le document et l'utilisateur. Pour les documents importants de nombreux professionnels interviennent dans leur réalisation.

Ainsi, la réalisation d'un document multimédia, nécessite une organisation, une planification sans faille. En effet, les moindres dysfonctionnements entraînent des surcoûts difficilement gérables. Dans ces conditions il est nécessaire, avant d'entamer la production effective, d'avoir ce que l'industrie appelle des « dossiers de définitions » performants. Il s'agit, à partir de ceux-ci, de se représenter ce que sera le document, avant toute réalisation - de quelque nature qu'elle soit. Un domaine proche du multimédia - de ce point de vue là - est l'audiovisuel, plus simple par certains aspects. En effet, l'aspect développement informatique spécifique à l'interactivité ${ }^{2}$ 'intervient pas. Le scénario est une des pièces maîtresses du dossier de définition.

Toutefois, si en termes d'organisation de la conception et de la réalisation les modèles de l'audiovisuel sont en partie adaptables au multimédia, les spécificités interactives du multimédia et des supports associés dépassent les différents modèles

2. Le sens le plus juste donné au mot interactivité utilisé dans ce texte est celui défini par Nicole Pignier et Benoît Drouillat (2008) : «l'interactivité désigne les animations cinétiques des médias informatisés et leurs capacités à calculer l'intervention de l'utilisateur. ", défini aussi en d'autres termes par Jean-Louis Weissberg (2002). 
de scénarii audiovisuels ainsi que les documents de préparation de la réalisation (découpage et story-board), trop linéaires.

\subsection{Un scénario multimédia pour quels documents?}

\subsubsection{Le scénario aujourd'hui}

Aujourd'hui, plusieurs types de concepteurs de documents multimédias existent. Les concepteurs qu'il est possible de qualifier de « solitaires » et ceux qui travaillent en équipe. Les premiers ont tendance à s'affranchir de la scénarisation et les seconds sont obligés d'écrire un scénario comme l'a mis en évidence Nicolas Viéville (2003). Le scénario s'appuie principalement sur l'environnement et la charte graphique ainsi que sur ce qui est qualifié du modèle de l'interactivité, c'est-à-dire des fonctionnalités d'accession aux différentes pages du document - généralement la définition de menus et boutons - (Fournier, 2003). Ce modèle de l'interactivité permet de réaliser les interfaces (graphiques ou autres) du document multimédia.

Or, tous les modèles de scénario multimédia utilisés restent sclérosés par le lien hypertexte et les documents sont figés. Jean-Pierre Balpe écrivait déjà dans les années 1990: «Si l'ensemble des informations et le graphe de représentation sont fixes, l'hypertexte est un ensemble fini de parcours possibles prédéterminés, c'est-àdire de réorganisations de la topologie de surface des informations. C'est la solution «classique» la plus simple - mais aussi, dans la mesure où l'intelligence d'un dispositif peut être définie comme sa capacité d'adaptation à des contextes divers, la moins « intelligente » - celle massivement choisie pour la réalisation de quatrevingts dix-neuf pour cent des CD-ROM actuels » (Balpe 1997). Aujourd'hui, les capacités des réseaux haut débit ont complètement balayé les CD-ROM, mais cette structure par lien est bel et bien présente dans la majorité des sites internet et la capacité d'évolution du document est bien maigre.

Bien sûr, la gestion actuelle des interfaces permet l'ajout de fonctions dépassant largement le simple lien hypertexte, notamment par l'utilisation d'outils d'intégration multimédias qui permettent de surligner, de déclencher des actions par simple glissement de souris ou autres fonctionnalités. Dans un autre contexte, les sites collaboratifs sont des documents qui évoluent par l'action d'utilisateurs. Ceux-ci se trouvent être non plus des lecteurs ou des interacteurs ${ }^{3}$ mais des «co-auteursréalisateurs ». Au bout du compte, la capacité réelle d'auto-évolution du document générée par l'inventivité de l'auteur est très limitée.

Ainsi, aujourd'hui, le scénario de document multimédia en tant que produit du design multimédia (que cela soit le web design, le Game design ou d'autres types de documents) se rapproche de la description d'un spectacle de marionnettes à fil. Chaque fil (qui représente un lien, une fonction prédéfinie), autorise une action

3. L'interacteur est le lecteur d'un document interactif. 
spécifique commandée par le marionnettiste (qui représente l'utilisateur). L'auteur agit alors en maître du monde : rien ne peut se faire, se prévoir, sauf si cela a été clairement imaginé par le concepteur. Nous sommes alors dans une logique en « rien-sauf » : rien n'est possible sauf ce qui a été expressément spécifié.

Si l'on veut représenter le document multimédia dans sa globalité en y intégrant la dimension de générativité et d'évolutivité du document, il faut balayer cette représentation hiérarchisée en adoptant un autre mode de fonctionnement.

\subsubsection{La diégèse comme scénario multimédia}

En fait, plus que de scénario, il serait judicieux de parler de potentiel scénaristique, c'est-à-dire de diégèse - pour reprendre un terme cinématographique. Cette notion, introduite par Étienne Souriau (1953), peut se définir comme tout ce qui appartient, dans l'intelligibilité du document, au monde supposé ou proposé par le récit. La diégèse est donc un monde virtuel, peuplé d'entités et régi par des lois internes. Les entités sont des objets, choses ou personnages réels ou imaginaires, qui participent à l'évolution et à la description de l'environnement.

En effet, contrairement à un scénariste audiovisuel qui se représente le déroulement linéaire du film, il est impossible à l'auteur multimédia de connaître l'organisation des unités d'informations qui seront perçues lors de la consultation du document. Ceci constitue un frein à la conception de documents génératifs ou évolutifs d'auteur.

Les possibilités logicielles actuelles peuvent permettre un raisonnement en automates dans la conception des documents. L'auteur définit alors chaque entité espaces (lieux concrets, abstraits...), objets (actifs, inactifs...), personnages (réels, imaginaires...) et autres - du document

Chaque entité «connaît » les actions, les modifications qu'elle peut engendrer. À l'inverse d'un raisonnement en «marionnettes à fil » - où l'auteur observe en maître du monde les différentes parties du document - le raisonnement "en automates » permet l'observation de l'environnement depuis chacune des entités. Il est alors possible d'imaginer le mode de fonctionnement de chaque entité en fonction du contexte. Les auteurs pourraient même s'orienter vers un raisonnement en «toutsauf » : tout est possible sauf ce qui a été interdit par l'auteur.

Toutefois pour adopter ce raisonnement, un changement d'état d'esprit radical s'impose. La représentation d'entités autonomes conduit à abandonner les représentations linéaires traditionnelles (la structure arborescente des scénarii) au profit d'une structure que les informaticiens pourraient qualifier d'agents intelligents (Ferber, 1995). La création d'agents intelligents repose sur la modélisation orientée objet (Meyer, 2000). Sans entrer dans le détail, chaque objet informatique reprend toutes les caractéristiques de la classe dont il est issu avec des spécificités complémentaires. Ainsi, s'il existe une classe «chien » et que l'on définit un objet «Bill», celui-ci hérite de toutes les propriétés de la classe chien (animal à 4 pattes, aboyer, bondir...) en précisant notamment que sa race est « cocker à poil roux ». 
En fin de compte, représenter le scénario sous la forme d'un diégèse revient à considérer le scénario comme une représentation objet du document - au sens informatique.

\subsubsection{Forme de représentation objet du scénario multimédia}

$\mathrm{Ce}$ changement de mentalité pose de gros problèmes idéologiques. Jean-Pierre Balpe dirait que «Le livre est tout le problème...» (Balpe, 2001). En effet, aujourd'hui, l'auteur (ou le concepteur) n'accepte pas que la scénation ${ }^{4}$ ne soit pas strictement équivalente à ce qu'il a prévu. Ceci limite obligatoirement le champ des possibles. Cet état d'esprit est tout à fait compréhensible : si l'auteur ne peut se représenter, par partie, toutes les scénations possibles du document, il se sent dépossédé de sa création. C'est un des problèmes majeurs auxquels sont confrontés les chercheurs qui travaillent sur la narration générative et interactive : quelle est la place de l'auteur dans une génération automatique de documents (textes, dialogues, images de synthèse...) (Szilas, 2005) ? Pourtant, l'auteur a une place prépondérante au sein des documents à entités autonomes. Il imprègne de sa vision créatrice le document, à travers toutes les descriptions des entités, de leurs évolutions possibles... Si le concepteur a défini le mode de fonctionnement des entités du document avec suffisamment d'inventivité, le document sera alors imprégné de sa force créatrice. L'auteur ne sera pas dépossédé de son œuvre, bien au contraire.

\subsection{Caractéristiques du scénario multimédia}

Le scénario, représentation abstraite du document, est le texte du document - au sens de (Gardies et Bessalet, 1995) précisé par Sylvie Leleu-Merviel (1996) : «la représentation abstraite du document est appelée le texte : elle utilise un formalisme de représentation adapté ».

Le «scénario » du document multimédia est donc un modèle du document à réaliser. Il intègre des fonctions d'interactivité.

Le scénario va permettre à tous les intervenants de la «préproduction » et de la réalisation du document de se le représenter sans qu'aucune partie du document n'ait été réalisée.

Au premier abord, le scénario permet d'évaluer les coûts de production et par làmême sa faisabilité financière. Dans un contexte de document de commande, il permettra de (ré)viser à la hausse ou à la baisse la dimension du document final. Pour un auteur, tout comme dans l'audiovisuel, le scénario peut permettre de trouver des financements pour la réalisation du document.

4. La scénation est l'ordre dans lequel les différents fragments du document sont perçus par le lecteur (Colin, 1992). 
Un scénario, tout comme n'importe quel dossier de définition, est manuscrit, ou du moins indépendant de tout processus d'industrialisation et de production.

\subsubsection{Indépendance du scénario vis-à-vis de la réalisation}

Le scénario est un document nécessaire à l'équipe de «préproduction»- ou l'équipe d'industrialisation - pour préparer la réalisation. Il permet d'élaborer les solutions techniques à mettre en œuvre pour réaliser le document. Si le scénario exprime clairement les choix communicationnels du produit et la forme perceptible qu'ils prendront, l'équipe de réalisation peut alors développer au mieux le document. Ainsi, le produit a plus de chance d'approcher les objectifs initiaux. Cela n'est envisageable que dans la mesure où le scénario est détaché de tout processus de production. En effet, même si le cahier des charges définit certaines contraintes techniques, elles ne doivent pas imposer un mode de création sclérosé.

L'auteur imagine son document multimédia. Il conçoit bien une œuvre interactive et, par là même, est guidé par ses représentations personnelles, par ses connaissances (ou ses méconnaissances) sur les possibilités techniques du support. Toutefois, le scénario ne se préoccupe pas de la réalisation : il s'affranchit de décrire des solutions techniques.

L'indépendance du scénario vis-à-vis de la réalisation implique qu'il se situe à un niveau conceptuel: le scénario n'est pas une simulation mais une représentation formalisée de l'œuvre (Colin, 1992). Le scénario se trouve donc éloigné de toute implantation informatique permettant une automatisation du processus de production. Il sera le vecteur de communication privilégié de l'équipe de réalisation. Chacun à son niveau possède ainsi une représentation mentale correcte du document, fondée sur la référence commune que constitue le scénario. Celui-ci expose les objectifs communicationnels généraux du document, mais aussi ceux des objets le composant.

Dans la mesure où cette représentation du document sert d'élément de référence pour son processus de réalisation, elle requiert un formalisme, un code, qui lui permet d'être comprise par le plus grand nombre d'intervenants au cours de la réalisation. Ce code s'identifie à un modèle formel de conception qui deviendra un outil favorisant la formalisation des idées du créateur. Il permet au scénario ainsi généré de souligner la cohérence structurelle de l'œuvre, avant d'aborder sa réalisation.

En conséquence, le modèle formel de conception est là pour permettre au créateur d'exprimer :

- clairement les fonctions des éléments du scénario,

- les solutions directement ou indirectement perceptibles par le lecteur, afin d'atteindre les objectifs communicationnels, au cours de l'élaboration du scénario lui-même.

Cela permet une vérification, en comparant les solutions proposées par le créateur à travers le scénario et les objectifs définis dans le cahier des charges 
fonctionnel. De plus, chacun des intervenants, imprégné de l'essence du projet, peut ainsi agir en adéquation avec les fonctions définies. Il s'agit alors pour lui de proposer les solutions techniques optimales dans le cadre de la réalisation, tout en ménageant les éventuelles «trouvailles » créatives et/ou esthétiques. Cela permet à tous un travail plus serein pendant la production et cela facilite la tâche du créateur. C'est ce qu'évoque Bertrand Tavernier: «Quand je prépare un film, je passe un temps fou à peaufiner le scénario. Avec Jean Cosmos, nous avons écrit 17 versions de Conan... Après je suis libre, je sais où je vais, je peux laisser place à l'improvisation » (Raspiengeas, 1997). Ainsi, lorsque débute le tournage, tout est parfaitement défini; le réalisateur peut alors se consacrer entièrement à l'aspect artistique de son œuvre.

Afin d'atteindre ces objectifs, il est préférable que le modèle formel de conception tienne compte des processus humains, tant en amont pour la démarche créative, qu'en aval du point de vue du lecteur.

\subsubsection{Adéquation aux processus humains}

Les processus humains à prendre en compte lors de la conception multimédia recouvrent plusieurs aspects : la création, la transmission de la représentation du document et l'interaction, voire de l'interactivité, entre le document et ses lecteurs.

\subsubsection{Processus de création}

La création est une évolution, une série d'échanges permanents, entre des idées et leur formalisation. L'expression d'une idée entraîne sa conceptualisation. Il s'agit par la suite de la développer, de la décomposer : un élément scénaristique vague se précise, s'affine. Le produit de cette formalisation engendre de nouvelles idées qui se doivent de respecter la cohérence du projet. Le mode de construction créatif passe-til constamment d'une spécification générale à une expression de détails et vice versa. À tous les niveaux, la possibilité d'une vision d'ensemble, ainsi que la précision des détails sont importants.

Un modèle scénaristique générique peut faciliter la décomposition d'entités signifiantes en plusieurs autres de taille inférieure ; cette décomposition d'éléments précise les entités initiales. Inversement, un auteur compose aussi son scénario à partir d'idées de détails. Il les intègre alors au modèle de conception général avant même que celui-ci ne soit complètement structuré. Il s'agit, en quelque sorte, d'une synthèse d'éléments scénaristiques. Cette synthèse s'appuie sur une composition d'éléments pour en introduire de plus généraux.

De plus, certains éléments de détail permettent au créateur de générer d'autres éléments scénaristiques - de détail ou plus généraux - tout en conservant la cohérence globale du document futur. Souvent, un point particulier est générateur de cohérence globale dans la mesure où ce détail sert de catalyseur à une partie de la démarche. Par ailleurs, les détails peuvent être des nœuds dramatiques, c'est-à-dire des étapes-clés structurantes et justifiant l'évolution générale du scénario. 
Ceci est conforme à la pensée de John Locke (1960). Pour lui, toutes nos idées proviennent de l'expérience: "Les observations que nous faisons sur les objets extérieurs et sensibles, ou sur les opérations intérieures de notre âme, que nous apercevons, et sur lesquelles nous réfléchissons nous-mêmes, fournissent à notre esprit les matériaux de toutes ses pensées. Ce sont là deux sources d'où découlent toutes les idées que nous avons ou que nous pouvons avoir naturellement ».

\subsubsection{Visualisation microscopique et macroscopique}

Le mode de représentation du document à différents niveaux de précision est particulièrement important pour l'étude de la cohérence ou la compréhension des objectifs de fonctionnement - tant logiciels, cognitifs que psychologiques - du document. Par ailleurs, certains intervenants n'ont besoin d'avoir qu'une vision d'ensemble, des fonctions générales, du document. Inversement certains autres n'exploitent que des éléments précis du scénario. Ils désirent néanmoins s'imprégner d'une sensation globale afin d'appréhender le sujet, ainsi que disposer du développement des points qui les concernent plus particulièrement.

En résumé, un modèle de scénario multimédia doit permette d'éclater des éléments généraux du scénario en composantes élémentaires et, inversement, de construire des ensembles généraux par assemblage d'éléments simples. Ces fonctions sont rattachées tant au scénario lui-même qu'à son modèle formel. En effet, la composition-décomposition d'éléments microscopiques et macroscopiques, sont aussi importantes pour l'écriture du scénario que pour sa lecture a posteriori.

\subsubsection{Autonomie des entités et modèle du lecteur}

Dans le domaine des jeux vidéo, l'autonomie des entités se définit à travers des règles de fonctionnement, des règles du jeu. Il est même couramment admis que les jeux ne sont pas scénarisables (Avarez et al., 2007). Ceci n'est qu'une des conséquences de l'absence de représentation en «automates». Le scénario va donc définir un certain nombre d'entités qui intégreront leurs règles de fonctionnement. Ainsi, chaque entité pourra interagir avec n'importe quelle autre entité. Le lecteur en étant une prépondérante.

Dans la mesure où le document est conçu pour un lecteur, la composante humaine du récepteur intervient pendant tout le processus de conception. Il est donc préférable que le scénario porte l'empreinte des relations, tant cognitives que psychologiques, voulues par l'auteur. Ceci peut passer par un modèle du lecteur.

Un modèle du lecteur a la charge de donner l'image que le concepteur se fait de l'utilisateur. Il ne s'agit pas de réifier le lecteur mais de spécifier au sein du document futur une réactivité s'appuyant sur un récepteur hypothétique. Ainsi, les évolutions du document pourront être déterminées par l'état imaginé du lecteur à travers son modèle. Il permettra en outre de définir arbitrairement un modèle mental du lecteur face aux messages perçus afin de faciliter : 
- la tâche d'évaluation,

- la compréhension des objectifs par tous.

De plus, un modèle du lecteur prendra en compte les modes d'action de l'utilisateur. Ces modes d'action, en conjonction avec les règles de fonctionnement du modèle mental, hypothétiques, autoriseront la définition d'une typologie de lecteur en fonction de sa réactivité et du document lui-même. Le document pourra alors élaborer une stratégie d'évolution adaptée aux différents types de lecteurs avec lesquels il échange. Il est à noter que quelques rares documents comme - déjà en 1995 - le CD-ROM «Le marché de Jean-Pierre COFFE » (Coffe, 1995) - se sont appuyés sur la détermination d'un caractère hypothétique de lecteur suivant son comportement et son attitude pendant la lecture.

Le lecteur fait partie intégrante d'un système dans lequel le document prend place avec son environnement technique. Le modèle du lecteur permet au document de se faire une image du récepteur humain en fonction des réactions de celui-ci perçues à travers « l'interface homme-machine ». L'image du lecteur au sein du document n'est pas une représentation exacte. Elle peut même s'avérer complètement fausse, éventuellement parce que le lecteur agit pour tromper le document, ou plus simplement parce que le modèle n'est pas adapté au caractère du lecteur. Dans un contexte créatif, le modèle du lecteur est généralement inventé de toutes pièces sans référent particulier; il est alors probable que la représentation du lecteur construite par le document ne corresponde pas à la réalité, elle traduit alors une démarche artistique. C'est cette démarche qui construira la cohérence du document vis-à-vis du projet de l'auteur.

\subsection{Fonction du scénario multimédia et de son modèle générique}

En résumé, les spécifications propres à un modèle générique de scénario multimédia sont classées sous deux rubriques. La première définit le scénario et son modèle à un niveau conceptuel et par la même indépendamment de l'étape de réalisation afin :

- de permettre un réel processus de création,

- d'aider le créateur à formaliser et développer ses idées,

- de permettre la production d'un scénario qui facilite la communication entre les intervenants.

La seconde recommande l'intégration de caractéristiques communicationnelles avec un être humain par le biais :

- d'un modèle de formalisation des fonctions,

- d'un modèle hypothétique du lecteur,

- d'un formalisme qui permette une appréhension du document depuis un point de vue macroscopique jusqu'à un point de vue microscopique et vice versa. 
Ces critères, qui peuvent paraître des critères de bon sens, ne sont que partiellement utilisés lors de la conception des documents multimédias. L'absence d'outil d'aide à l'écriture, et en amont, l'absence de formalisation de la représentation abstraite des documents multimédias au sens large (des sites internet aux mondes virtuels en passant par les jeux vidéo) sont une des raisons de la sclérose des documents évoquée précédemment.

La suite du document propose une structure d'écriture pour les auteurs de documents multimédias qui s'appuie sur les principes qui viennent d'être définis.

\section{Scénarisation moléculaire}

Le principe général de la représentation moléculaire s'appuie fortement sur la diégèse du document. La représentation en entités permet de formaliser des éléments évolutifs et/ou génératifs. Ainsi, un modèle du lecteur, permet d'imaginer des modalités d'interaction entre les interacteurs et le document, et peut - en outre - être défini par hypothèse.

\subsection{Introduction à la scénarisation moléculaire}

L'objectif avoué du modèle moléculaire est de supporter l'écriture et la représentation de documents dynamiques tout en ne permettant aucune automatisation de la réalisation à partir du scénario. Il s'agit de fournir un formalisme que n'importe quel auteur, n'importe quel artiste, hermétique aux outils techniques de réalisation, puisse exploiter pour écrire le scénario d'un document. Ce scénario lui permettra de faire comprendre et de faire réaliser les documents multimédias qu'il a imaginés.

Ce modèle, comme le sous-entend son appellation, a été conçu par analogie avec des notions de chimie et s'appuie donc sur des atomes et sur des molécules.

Le modèle moléculaire ne considère plus le scénario comme une succession de choses, de faits et d'actions. Il s'agit de représenter des entités, avec leurs règles de fonctionnement, leurs actions potentielles en fonction de l'environnement.

Les atomes et molécules scénaristiques représentent, décrivent, les entités, leurs actions potentielles induites par des circonstances ou conjonctures particulières. Ils sont nécessaires à la représentation formalisée du document.

Les atomes sont des éléments simples. Ils peuvent être décrits par une entité textuelle unique. A l'inverse, les molécules sont des éléments complexes dont la description nécessite plusieurs éléments distincts. Ainsi, une molécule peut être composée de plusieurs atomes ou molécules. 


\subsection{Atomes et molécules d'un scénario}

Il y a 3 grandes familles d'atomes et molécules pour constituer le modèle :

- les atomes et molécules diégétiques, décrivant les entités du document ;

- les atomes circonstanciels et les molécules conjoncturelles, décrivant les différentes circonstances intervenant dans le document ;

- les atomes et molécules d'actions, décrivant les actions faisant évoluer les situations, les entités, l'état du document.

\subsubsection{Atomes et molécules diégétiques}

Les atomes et molécules diégétiques représentent des entités du document.

Chaque atome ou molécule diégétique est un fragment de scénario. Il représente l'élément constituant d'une entité $d u$ document. Un atome diégétique est conceptuellement et complètement décrit par un élément structurel unique.

Un atome diégétique est une représentation élémentaire (partielle ou totale) d'une entité du document au sein du scénario.

Cet élément est suffisant pour définir conceptuellement - à une étape particulière de la conception - la partie de l'entité concernée.

Les atomes diégétiques sont figurés ici par des éléments ovales.

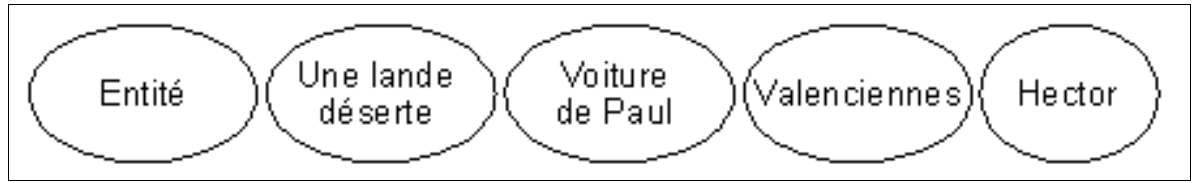

Figure 2. Atome diégétique et exemples

Une molécule diégétique est la représentation conceptuelle complexe, d'une entité autonome du document.

Une molécule diégétique étant la représentation d'une entité du document, la dimension temporelle des molécules est sous-jacente (les entités ont la capacité d'obéir à des règles de fonctionnement ou de comportement, et sont ainsi liées au temps).

Une molécule diégétique est composée, outre du nom qui la définit :

- de facteurs d'enclenchement: ils définissent les conditions d'occurrence de l'entité représentée,

- de descriptions perceptibles : elles décrivent les éléments influant sur la perception physiologique de l'entité par le lecteur, 
- de descriptions sémantiques : elles décrivent tout ce qui n'est pas d'ordre perceptible.

Le nom donné à l'entité permet de l'identifier. Cet identificateur correspond généralement à une expression représentative de l'entité en question. Ce nom peut aussi être qualifié d'alias.

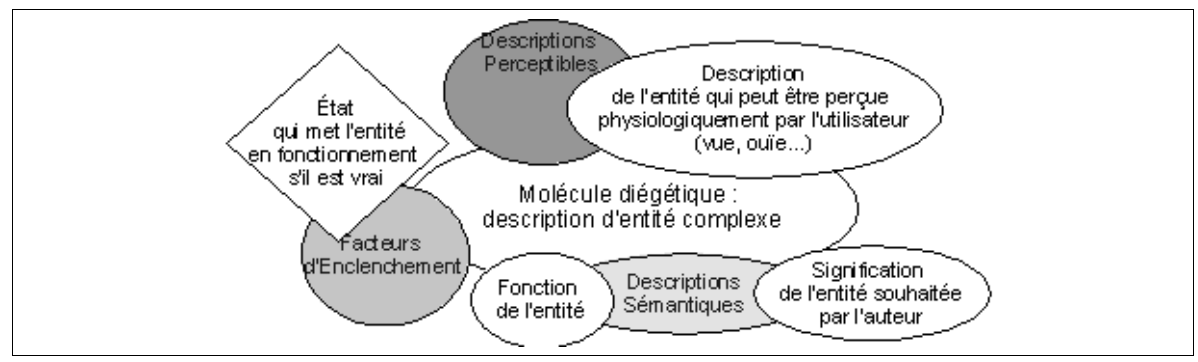

Figure 3. Représentation graphique d'une molécule diégétique

Les éléments de descriptions perceptibles (DP) décrivent les caractéristiques de l'entité concrètement perceptibles. Cette description peut contenir un ensemble d'atomes ou de molécules à caractère sensible ${ }^{5}$; c'est-à-dire liés à des manifestations qui toucheront physiologiquement nos sens de perception (si les entités correspondantes sont affichées). Ceux-ci peuvent être comparés à la description des «manifestations d'un message » en sémiotique.

Ainsi, les descriptions perceptibles définiront ce qui peut être vu et entendu concrètement de l'entité au cours de la lecture du document. Ces descriptions peuvent être purement factuelles (définition d'un état de fait) ou définir des actions potentielles de l'entité (déplacements, modification de l'environnement...).

$\mathrm{Si}$, par exemple, une molécule diégétique représente une maison, les dimensions et l'aspect extérieur de la bâtisse constituent des descriptions perceptibles.

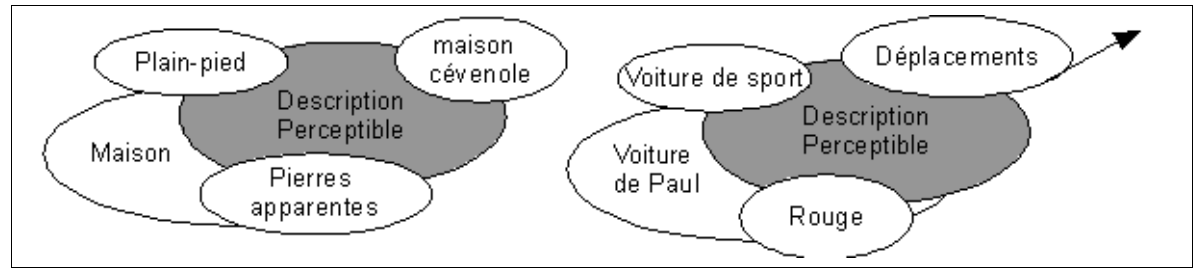

Figure 4. Exemple de descriptions perceptibles de 2 molécules

5. Sensible est considéré dans son sens passif: qui peut être perçu par les sens (ROBERT, 1989). 
Les éléments de descriptions sémantiques (DS) précisent les caractéristiques du signifié de l'entité et plus généralement de tout ce qui ne peut être directement perçu physiologiquement.

Le sens voulu par l'auteur en concevant l'entité fait partie des descriptions sémantiques. Les fonctions de communication de l'entité sont aussi décrites, le cas échéant, dans les descriptions sémantiques.

$\mathrm{Si}$ par exemple l'auteur souhaite définir une molécule représentant un personnage qui a peur, l'indication de la peur du personnage relève de la description sémantique.

À ce niveau de la conception, cette peur n'est pas observable directement. Cela n'est du reste pas nécessaire. Cette description sera convertie par des faits perceptibles, (gouttes de sueurs, yeux exorbités, immobilité, tremblements nerveux, cris, etc. qui relèvent de la description perceptible) beaucoup plus tard - lorsque l'on tend vers la scénique ${ }^{6}$ et la fabrication du document.

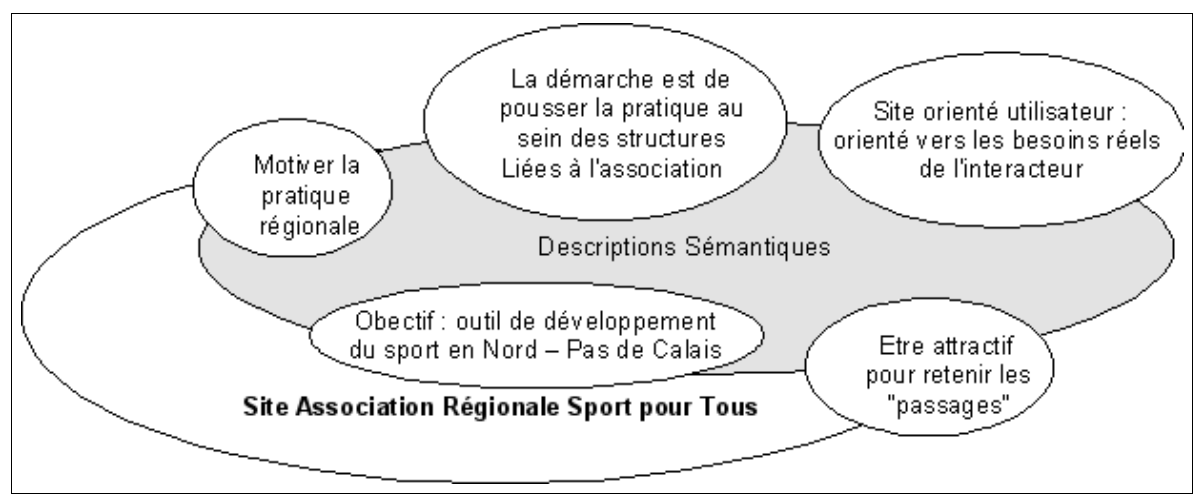

Figure 5. Exemple de descriptions sémantiques liées à des fonctions du document

Les descriptions sémantiques permettent aussi de définir certaines indications utiles à la mise en scène du document. Ainsi, la description psychologique d'un personnage s'effectue au sein de cette description.

Dans les premières étapes de l'écriture du scénario, au sein des macromolécules (molécules les plus générales), les descriptions sémantiques sont très importantes. Elles permettent d'indiquer la fonction (particulièrement en termes de signifié) des documents en cours d'écriture.

6. La scénique désigne le processus permettant de transposer le texte en une réalité concrète : elle résulte de choix esthétiques, de contraintes pratiques ou financières... (LeleuMerviel, 1996). 
Le sens indiqué par ces descripteurs est potentiel. En effet, les entités décrites n'existent pas lors de la conception (sauf en cas d'utilisation d'éléments d'archives). Les descriptions sémantiques constituent une note d'intention auprès des différents intervenants. Elles contiennent l'objectif du sens des informations transmises au lecteur.

Les facteurs d'enclenchement (FE) mettent en fonctionnement l'entité. Suivant l'entité décrite, il s'agit - si l'une des circonstances (ou conjonctures) spécifiant les facteurs est vraie - de l'afficher, la mettre en scène, la rendre active...

Ces facteurs d'enclenchement sont un des acteurs majeurs de l'autonomie des entités représentées. En effet, ce sont les entités elles-mêmes qui contiennent l'information des conditions nécessaires (mais pas forcément suffisantes) de leur mise en fonction (affichage, arrivée dans l'espace de perception de l'interacteur...).

Fescriptions perceptibles

Figure 6. Exemple de facteur d'enclenchement sur une molécule de jeu dans une représentation tabulo-graphique

On peut noter qu'une entité active n'est pas forcément perçue directement par l'interacteur. Si par exemple, une ampoule s'allume dans une boîte fermée de façon hermétique, celle-ci n'est pas perçue comme étant en fonctionnement.

\subsubsection{Atomes circonstanciels, molécules conjoncturelles}

Un atome circonstanciel, formalisé à la figure 7 par un losange, est la représentation scénaristique d'une circonstance ${ }^{7}$.

Une circonstance spécifie une occurrence d'état. L'état d'une circonstance est évalué par une variable logique. Ainsi, en logique binaire, une circonstance est vraie ou non vraie ${ }^{8}$.

7. Circonstance : particularité qui caractérise une configuration déterminée constituant l'une des modalités conditionnelles d'occurrence d'un événement. (Leleu-Merviel, 1996).

8. Il est tout à fait envisageable d'adopter une logique floue (Zadeh, 1965) pour améliorer l'évolutivité du document. 
62 RSTI - DN - 12/2009. Conception, design des documents numériques

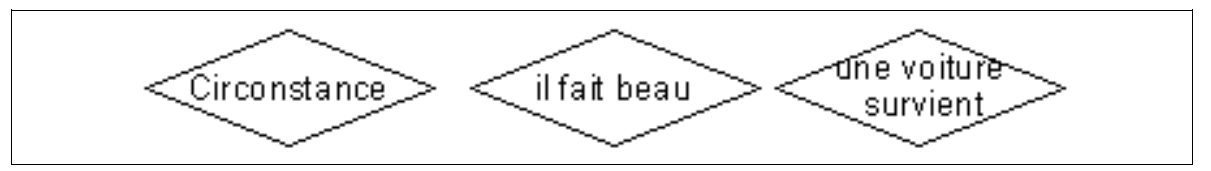

Figure 7. Atome circonstanciel et exemples

Si une circonstance se révèle insuffisante pour décrire un état, celui-ci sera décrit par une conjonction de faits, c'est-à-dire une conjoncture.

Une conjoncture, entité complexe, est représentée par une molécule conjoncturelle (cf. figure 8). Une molécule conjoncturelle est la représentation d'une conjoncture $^{9}$ au sein du scénario.

Une conjoncture est un ensemble de circonstances - ou de conjonctures, constituant une proposition logique. Comme pour une circonstance, une conjoncture est évaluée conformément à la logique retenue (binaire, floue...), même si, pour simplifier le propos la logique binaire a été retenue ici.

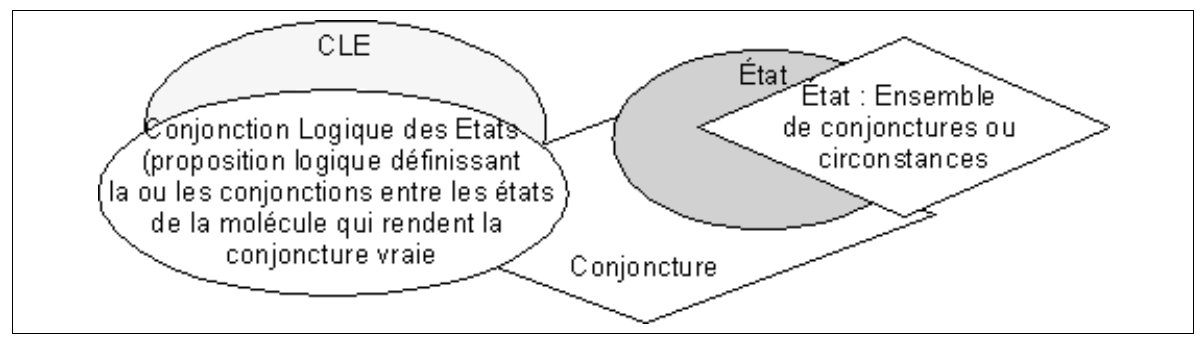

Figure 8. Représentation graphique d'une molécule conjoncturelle

Une molécule conjoncturelle est composée :

- d'états qui correspondent à des circonstances ou à des conjonctures qui seront vérifiés pour rendre la conjoncture vraie,

- de conjonctions logiques des états qui définissent les liens logiques entre tous les états de la molécule.

Les États d'une molécule conjoncturelle sont des faits que la molécule conjoncturelle contient. Ils représentent des circonstances, conjonctures qui contribuent à rendre la conjoncture vraie.

9. Conjoncture : état particulier qui résulte d'une conjonction de circonstances données, tel que cette conjonction soit considérée comme le point de départ d'une évolution ou d'une action. (Leleu-Merviel, 1996). 
Une molécule conjoncturelle peut être définie par un état unique. Dans un scénario, cela permet de réexprimer différemment un état. Cela permet de définir plus précisément un fait, potentiellement déclencheur d'une action, d'une entité, sans alourdir la molécule qui contient cet état. La figure 9 en est un exemple.

\begin{tabular}{|l|l|}
\hline \multirow{2}{*}{$\begin{array}{l}\text { Rotation } \\
\text { autorisée }\end{array}$} & \multicolumn{2}{|l|}{ Conjonction Logique des Etats } \\
\cline { 2 - 3 } & Etats \\
\cline { 2 - 2 } & Aucune partie d'une des cases composant la forme n'est en contact avec le décor \\
\hline
\end{tabular}

Figure 9. Exemple de conjoncture définie par un état unique

Lorsqu'une molécule conjoncturelle contient plusieurs circonstances ou conjonctures, des règles sont nécessaires pour hiérarchiser leur fonctionnement. $\mathrm{Ce}$ sont le rôle des conjonctions logiques des états.

Les conjonctions logiques des états (CLE) définissent les liens logiques entre tous les états qui définissent la molécule conjoncturelle et contribuent à son expression.

En effet, une conjoncture peut être vraie si une seule des circonstances spécifiées l'est ; inversement, il peut y avoir la conjonction obligatoire d'un ensemble de circonstances pour la vérifier. Ces conditions définissent donc des relations du type « et », « ou », « non » et autres opérateurs logiques, entre les éléments contenus dans la conjoncture.

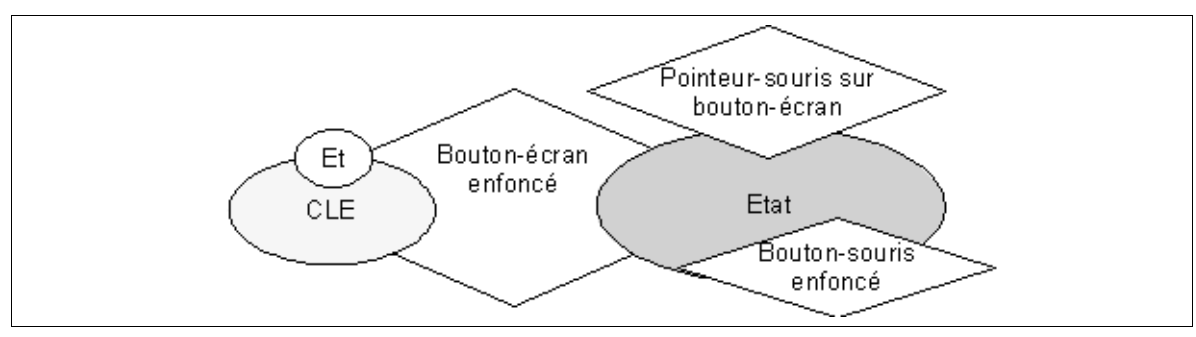

Figure 10. Exemple de description de conjoncture

A un temps $t$ donné, une circonstance ou une conjoncture est vraie ou non-vraie (c'est-à-dire fausse ou indéterminée). Suivant leur valeur, des entités pourront être mises en fonctionnement (en fonction des facteurs d'enclenchement des molécules diégétiques correspondantes), ou des événements pourront être déclenchés. 
Il est intéressant de noter que, malgré la logique binaire utilisée, il est possible à l'auteur d'introduire une forme de logique floue. Par exemple si une molécule est considérée comme vraie si «le personnage est proche du piège » et si l'auteur n'a pas défini de critère absolu de proximité, le programmeur peut adapter cette notion en logique floue.

\subsubsection{Atomes et molécules d'actions}

Le dernier type d'atome, l'atome d'action - qui prend, à la figure 11, la forme d'un ovale terminé par une flèche - définit un événement du scénario.

Un atome d'action représente un événement élémentaire. Un événement est une action - ou un ensemble d'actions - visant à faire évoluer le système.

Les actions induites peuvent se limiter à une modification de la scénation, c'est-à-dire à des actions d'affichage. Cependant, si les molécules d'action sont des éléments du scénario d'un document à données génératives, certaines d'entre elles définissent des opérations sur le contenu d'atomes ou molécules diégétiques. Les événements permettent donc d'induire la modification ou la création d'entités. Un événement produit une action sur l'environnement. La situation générale du système s'en trouve modifiée.

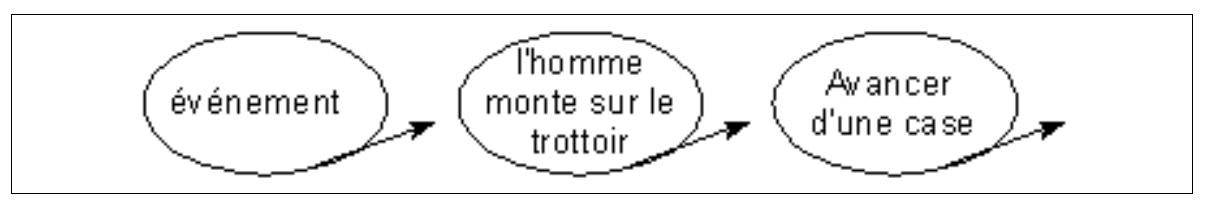

Figure 11. Atome d'action et exemples

Une molécule d'action (figure 12) est composée

- de facteurs d'enclenchement,

- d'actions, qui sont les différents événements composant la molécule,

- de conjonction logique des actions qui établissent un lien logique entre les différentes actions.

Les facteurs d'enclenchement sont l'ensemble des conjonctures et/ou des circonstances qui, lorsqu'elles sont vérifiées, provoquent l'événement.

Les facteurs d'enclenchement ont strictement le même rôle au sein des molécules d'action qu'au sein des molécules diégétiques. Si le facteur d'enclenchement est vrai, alors l'action est engendrée.

Les Actions sont les événements induits par la validation de l'événement. Ils peuvent être de typologies diverses. Ainsi, les événements peuvent définir la transformation ou la création d'entités. 


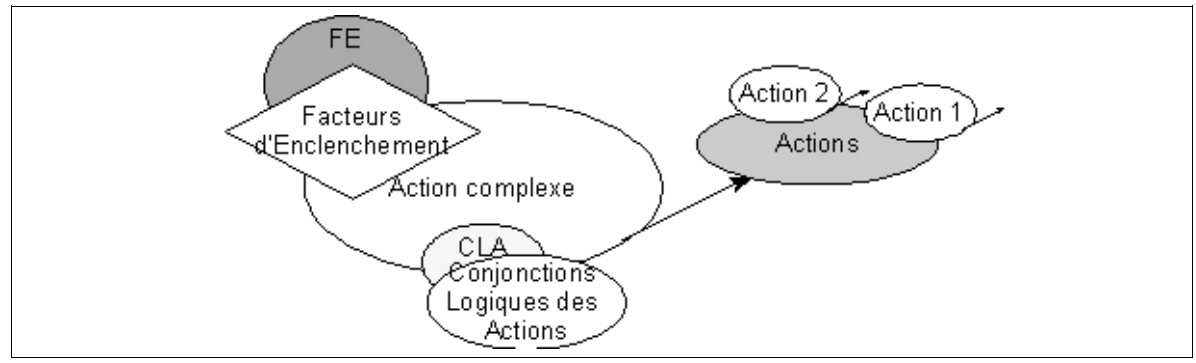

Figure 12. Molécule d'action

Les conjonctions logiques des actions (CLA) définissent les opérateurs logiques régissant les actions contenues dans l'événement. En effet, un événement se caractérise éventuellement par la conjonction de plusieurs autres événements. Il peut s'agir d'un enchaînement, d'une simultanéité ou même d'un choix entre plusieurs actions. Les conjonctions logiques des actions jouent un rôle de règles de gestion des événements contenus.

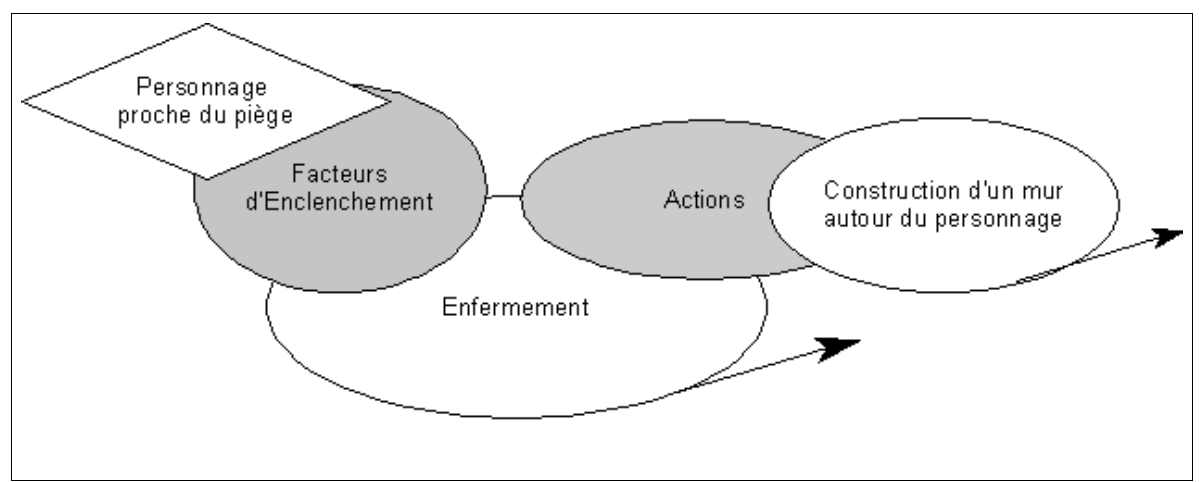

Figure 13. Exemple de molécule d'action

Ces opérateurs logiques intègrent par exemple l'algèbre d'ALLEN (Allen, 1986 ; Allen, 1994) ${ }^{10}$.

10. L'Algèbre d'ALLEN définit une temporalité entre 2 entités A et $B$ :

Avec des relations rigides

- A égale B (synchronisation parfaite),

- A démare $B$ ( $A$ et $B$ démarrent simultanément),

- A finit B (A et B se terminent simultanément), 


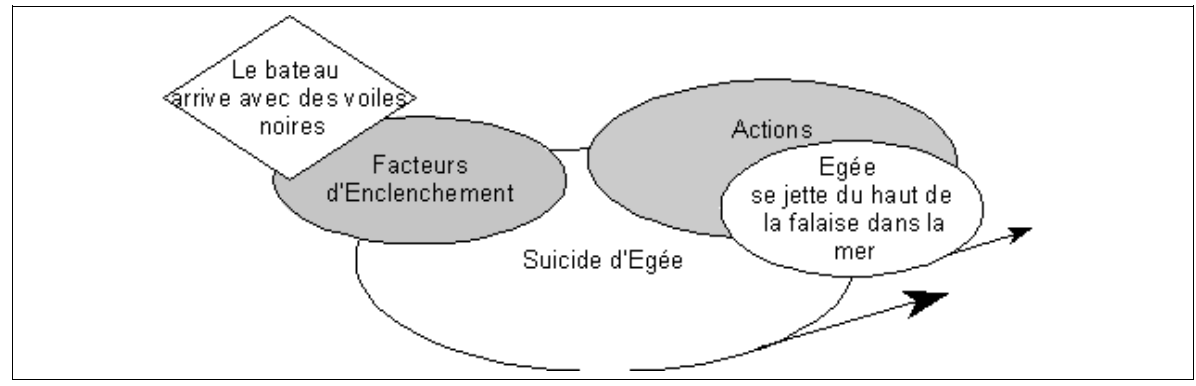

Figure 14. Autre exemple de molécule d'action

\subsection{Représentation des atomes et molécules}

\subsubsection{Formes de représentation}

À ce jour, de multiples représentations des atomes et molécules sont possibles. Trois exemples ont déjà été présentés précédemment :

- Une représentation graphique (majoritairement employée dans ce document),

- Une représentation textuelle,

- Un représentation tabulaire,

- Une représentation graphico-tabulaire,

N'importe laquelle de ces représentations est utilisable. Dans la mesure où la structure moléculaire est respectée, il est même possible d'utiliser sa propre représentation. Dans ce texte deux représentations sont utilisées : la représentation graphique et la représentation graphico-tabulaire. La première parce qu'elle est très ergonomique à la lecture pour bien différencier les différents types d'atomes et de molécules. Elle présente toutefois le défaut d'être difficile à utiliser par un auteur en absence de logiciel de «traitement de scénarii multimédias » ${ }^{11}$. La seconde est un bon compromis entre l'ergonomie d'écriture et la lisibilité. Un simple modèle de document de traitement de texte permet d'écrire le scénario.

- A rencontre B (B commence lorsque A a terminé, ce que l'on peut qualifier, si A et B sont des événéments d'événements séquentiels).

Et des relations flexibles

- A pendant $(t) B$ (A démarre $t$ après que $B$ ait démarré, avec $A$ se terminant avant $B$ ),

- $A$ avant $(t) B$ (A démarre $t$ après que $B$ ait fini),

- A recouvre ( $t$ ) $B$ ( $B$ démarre $t$ après que $A$ ait démarré, avec $B$ se terminant après $A$ ).

11. Par analogie avec un traitement de textes 


\subsubsection{Différentiation atome/molécule}

Dans un scénario, il est nécessaire de différencier une molécule d'un atome au sein d'une autre molécule. Dans la représentation moléculaire « graphique » adoptée, les molécules sont «ombrées », ce qui les distingue d'un atome. Dans le cas d'une utilisation graphico-tabulaire, il a été décidé d'utiliser le souligné avec une couleur différente (lorsque l'on travaille en couleur).

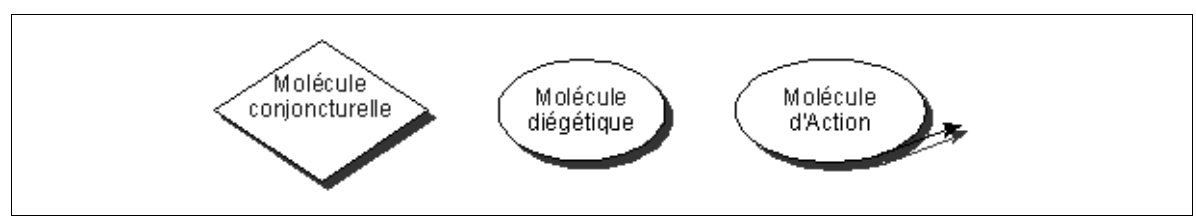

Figure 15. Molécules fermées

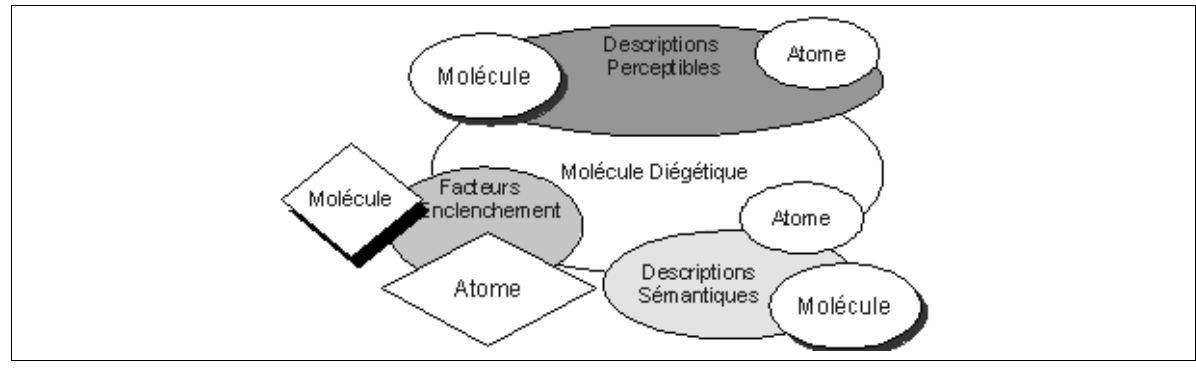

Figure 16. Différentiation atome/molécules dans une représentation graphique

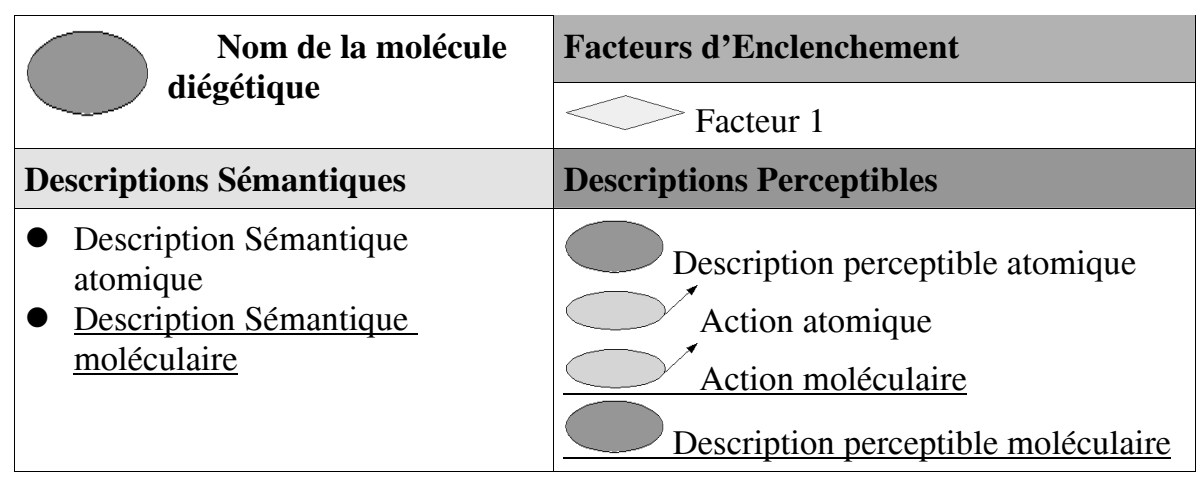

Figure 17. Différentiation molécules/atomes dans une représentation graphicotabulaire 
Ces modes de différentiation atome/molécule proviennent principalement des fonctionnalités d'hyperliens de certains logiciels généraux utilisés pour l'édition de texte ou de graphique. En effet, lors d'une lecture de la version informatique du document, la sélection d'une molécule située dans une autre molécule permet d'accéder à la représentation détaillée de la molécule en question.

La représentation symbolique standardisée des hyperliens dans du texte sont ce texte souligné avec une couleur différente (généralement le bleu). Cette représentation standardisée est ombrée sur des logiciels graphiques orientés « représentation de systèmes en automatique ».

\section{4. Écriture d'un scénario}

Le modèle moléculaire repose, avant tout, sur une formalisation des idées, sur l'élaboration d'un texte au sens littéral du terme. Ce texte représente le document aux yeux de l'auteur, c'est-à-dire le scénario.

Entre l'idée de document et le scénario terminé, de multiples étapes jalonnent le travail de l'auteur.

D’une manière générale, avant le travail d'écriture, les idées de l'auteur (ou du commandiataire dans un contexte industriel), sont les fonctions du document ${ }^{12}$.

\subsection{Fonctions du document et début de l'écriture}

Les fonctions du document s'expriment généralement à travers les descriptions sémantiques et perceptibles des molécules diégétiques. Le document, à ce stade est dans son état « intention ». Le document en lui-même est une entité. Il sera donc décrit par une molécule, que l'on pourra qualifier de molécule mère ou de molécule globale.

A l'état initial, la molécule mère contient les différentes intentions de l'auteur. Ses descriptions sémantiques permettent alors de définir les fonctions du document. Ainsi, l'auteur commence par exposer des idées, parfois très descriptives éventuellement limitées par sa propre imagination. Ces idées ne sont pas obligatoirement structurées. Ce n'est que par la suite, que l'auteur développe le scénario pour donner des solutions - au plan de la communication - aux idées exposées - qui relèvent des fonctions du document. La molécule mère - ou molécule globale - représente l'intégralité du document.

$\mathrm{Si}$ au début de l'écriture, la molécule mère ne contient guère que les fonctions du document, lorsque le scénario est terminé, la molécule globale contient l'intégralité des molécules et atomes représentant le document ${ }^{13}$.

12. Les fonctions sont les actions d'un produit ou de l'un de ses constituants exprimés exclusivement en termes de finalité (AFNOR, 1995). 
Progressivement, à partir des fonctions, l'auteur définit les entités ${ }^{14}$ qui structurent le scénario. Il a la possibilité de décomposer et d'identifier (donner des solutions au plan de la communication) des fonctions. Il a aussi la possibilité d'assembler, d'intégrer, des molécules représentant des entités précédemment rédigées. Ces fonctions du modèle moléculaire constituent les opérations structurelles de celui-ci.

\subsection{Opérations structurelles}

Progressivement, à partir des fonctions, l'auteur définit les entités qui structurent le scénario. Pour composer le scénario, développer ses idées, les organiser, l'auteur va pouvoir s'appuyer sur des opérations structurelles. Par exemple, un atome, préalablement défini, peut se révéler trop imprécis au cours de l'écriture. Cet atome scénaristique pourra alors être affiné. Sa décomposition permettra de produire plusieurs éléments (atomes et/ou molécules). Inversement il est possible de constituer une molécule « de synthèse » (au sens de l'opération intellectuelle). C'està-dire que plusieurs atomes ou molécules peuvent être regroupés afin de synthétiser un nouvel élément, des idées...

Les modalités de ces opérations utilisent notamment des propriétés de fission et de combinaisons atomiques.

La combinaison consiste à regrouper plusieurs molécules et/ou atomes afin de former une nouvelle molécule plus complexe.

Ainsi, la combinaison crée une molécule nouvelle en " assemblant» des atomes et des molécules existantes. Ce mode de conception est utile lorsque qu'il faut constituer des entités pour assurer la cohérence dramatique d'un document.

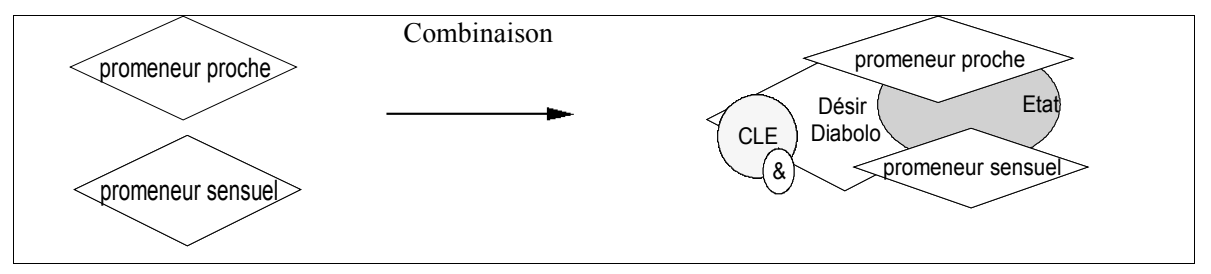

Figure 18. Combinaison moléculaire

Le processus de fission atomique permet de scinder un atome en plusieurs autres, comme dans l'exemple de la figure 19. La fission seule n'est que très rarement utilisée. Elle est utilisée généralement lors d'une décomposition.

13. Un exemple de molécule globale du jeu de Tétris a été donné à la figure 15.

14. Il est à noter que, dans certains cas, ces entités peuvent très bien n'avoir aucune représentation perceptible. 
Le processus de décomposition atomique permet de remplacer un atome par une molécule complexe, équivalente au plan général, mais plus précise.

Si la réalisation de combinaisons est importante, la décomposition d'atomes et molécules l'est tout autant. Il s'agit pour l'auteur, grâce à cette fonction, de préciser ses idées, ses entités. Il s'agit du processus traditionnel de création. De la définition d'une idée générale, on spécifie ce qui permet d'arriver à la description des éléments qui permettent de définir cette idée.

En phase terminale d'écriture (voire en phase de "préproduction ») certaines décompositions permettent d'améliorer la compréhension, ou du moins de réduire l'écart entre la représentation de l'entité que se fait l'auteur et celle comprise par l'équipe de réalisation. En effet, si le créateur a représenté une entité « oiseau » par un atome, il peut vouloir, après un certain temps de travail, décomposer la représentation de cet oiseau en caractérisant sa famille, la couleur de ses plumes ou de son bec afin qu'il représente parfaitement l'image mentale qu'il s'en fait.

Lors d'une décomposition atomique, tout se passe comme si l'on effectuait la fission d'un atome en plusieurs autres atomes de taille inférieure et qu'immédiatement après ceux-ci se combinaient afin de former une molécule.

Dans l'exemple qui suit, la décomposition de l'atome «voiture » conduit à la description de la molécule voiture. La fission de l'atome voiture conduit à deux atomes. La combinaison de ceux-ci aboutit à la synthèse de la molécule voiture.

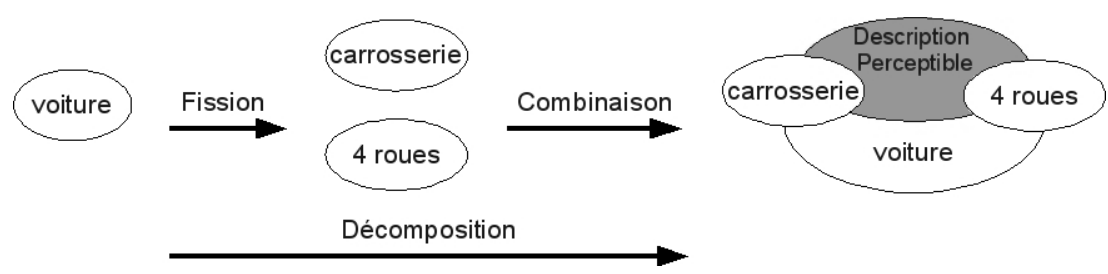

Figure 19. Fission suivie d'une combinaison

Un autre exemple, relatif à la conception d'un document sur l'état du monde, conduit à définir une molécule "Monde» à partir de plusieurs entités que sont les continents (figure 20). Chacun d'entre eux peut être découpé en vastes zones géographiques et/ou en états indépendants. Il s'agit alors d'effectuer une succession de fissions et de combinaisons afin d'arriver au niveau de détail scénaristique escompté. 


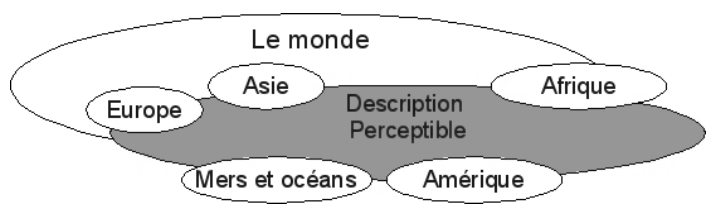

Figure 20. Molécule composée uniquement d'atomes

Il est à noter que lors d'une opération de décomposition, l'élément concerné devient «ombré» ou «souligné» (comme cela a déjà été précisé) afin de différencier les atomes des molécules (figure 21).

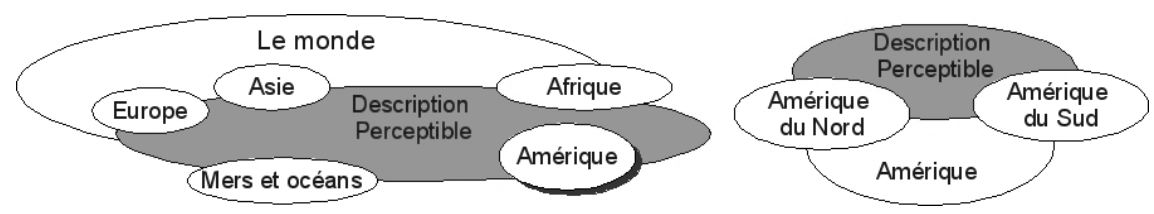

Figure 21. Résultat d'une décomposition de l'atome "Amérique »

Très utile lorsque 2 atomes ou molécules représentant la même chose ont le même nom, l'opération de substitution consiste à remplacer un atome ou une molécule par un nouvel élément. Il peut s'agir de substituer un atome par un autre de fonction similaire, mais il est possible de le remplacer par un élément dont la fonction est différente. En effet, il n'est pas rare qu'au cours du processus de création, certains points soient sérieusement modifiés. Par exemple :

- « véhicule automoteur léger à quatre roues permettant le transport de plusieurs personnes avec quelques bagages » substitué en « voiture»,

- «Château isolé » substitué en « grande demeure isolée ».

De façon plus simple, un auteur exprime rarement une idée sans la retravailler sous une forme ou sous une autre. La réécriture, la modification d'une phrase de scénario peuvent-être assimilées à une substitution.

\subsection{Orientation d'une scénation}

La scénation d'un document interactif est imprévisible. Cependant, le créateur peut introduire des orientations ou des critères de scénation à travers les facteurs d'enclenchements ou les conjonctions logiques. 


\subsubsection{Règles sur les facteurs d'enclenchement}

Les facteurs d'enclenchement sont des circonstances ou des conjonctures qui définissent les conditions pour lesquelles les entités ou les événements correspondants entreront en activité. Si un seul des facteurs défini au sein d'une molécule est vérifié, l'entité est validée ou l'événement se produit. Si la validation ou la mise en service - d'une entité est conditionnée par la conjonction ordonnée de facteurs, il suffit de créer une conjoncture appropriée.

Si une entité est en service, elle valide elle-même, et récursivement, les entités qui la constituent (à la condition que leurs facteurs d'enclenchement soient vérifiés) ; si la représentation de ces dernières n'a aucun facteur d'enclenchement, elles sont alors validées par défaut. Ainsi, pour que l'entité « docu» soit validée (cf. figure 22) il faut que le facteur d'enclenchement «à partir du début de séance » soit vérifié. La molécule diégétique ${ }^{15}$ " docu» est constituée de deux autres molécules traitant de «La vie des mygales en Génousie » et «Les ours de Poldavie ». Le premier thème est traité à l'aide d'un film documentaire «film 1 ». Aucune contrainte d'enclenchement n'est spécifiée pour «La vie des mygales en Génousie», par conséquent, cette entité constituée de « film 1 » est mise en service dès que « docu » est validée. Ce processus pourrait être qualifié de "validation automatique ». Par contre en ce qui concerne l'entité «Les ours de Poldavie », elle ne sera validée que lorsque le documentaire « film $1 »$ sera terminé.

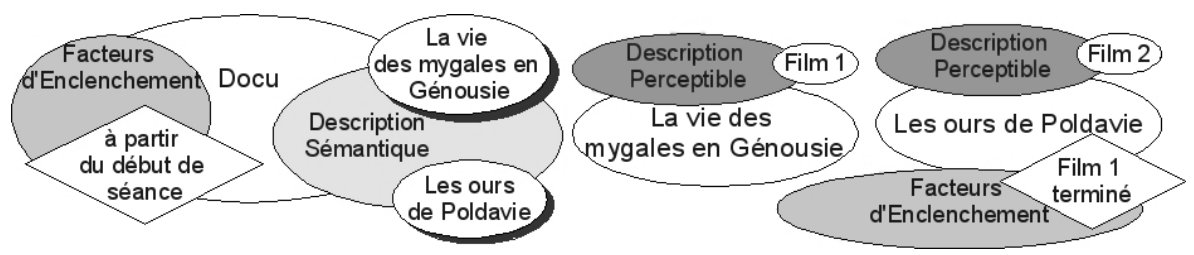

Figure 22. Exemple de représentation de validations «imbriquées 》

Une absence de facteurs d'enclenchement engendre la validation systématique des entités ou des événements, dès qu'un élément les contenant est en service.

\subsubsection{Opérateurs logiques de conjonction}

Les conjonctions logiques des états et des actions intégrées respectivement aux molécules conjoncturelles et molécules d'actions sont constituées d'opérateurs logiques. Ceux-ci définissent les conjonctions entre éléments constitutifs. Certains de ces opérateurs sont présentés dans ce texte, mais leur liste n'est pas exhaustive. Un

15. Pour alléger le discours, l'expression «molécule (diégétique, conjoncturelle ou d'action) représentant l'entité - ou l'événement, ou la conjoncture - X » est contracté en "molécule (diégétique, conjoncturelle ou d'action) $\mathrm{X} »$ ). 
auteur peut définir un nouvel opérateur logique si l'élément scénaristique développé le requiert.

Ainsi, des règles de priorités d'enclenchement peuvent être définies en s'appuyant sur des conjonctions logiques des états ou des actions. Si par exemple le créateur décide qu'une partie du document sera constituée d'une simulation de diaporama avec un accompagnement musical (cf. figure 23), il peut vouloir synchroniser précisément les entités visuelles et sonores. En ce cas, la molécule d'action «jouer diaporama 1 » est un exemple de scénarisation de cet extrait documentaire. Cette molécule précise que les événements «Jouer musique» et «afficher photographies» seront synchrones, ce qui demande une attention particulière lors de la réalisation. Par contre, la molécule «jouer diaporama 2 », en raison de l'utilisation de la conjonction logique '\&', indique que l'événement correspondant déclenchera « afficher photographies » et « jouer musique », mais que leur parfaite synchronisation n'est pas requise. La conjonction logique des actions "séq" de la molécule d'action "afficher photographies" indique que les trois photographies sont affichées les unes après les autres.

L'auteur, comme cela a été déjà abordé en section 3.2.3., peut aussi utiliser l'algèbre d'ALLEN.

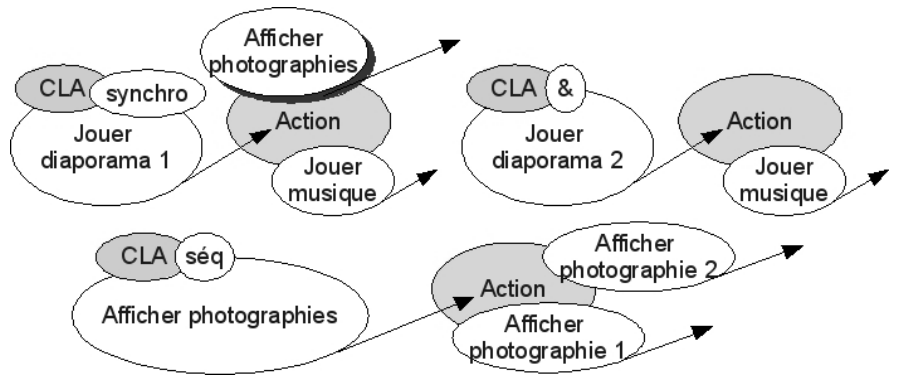

Figure 23. Exemple d'actions parallèles et séquentielles synchrones ou non

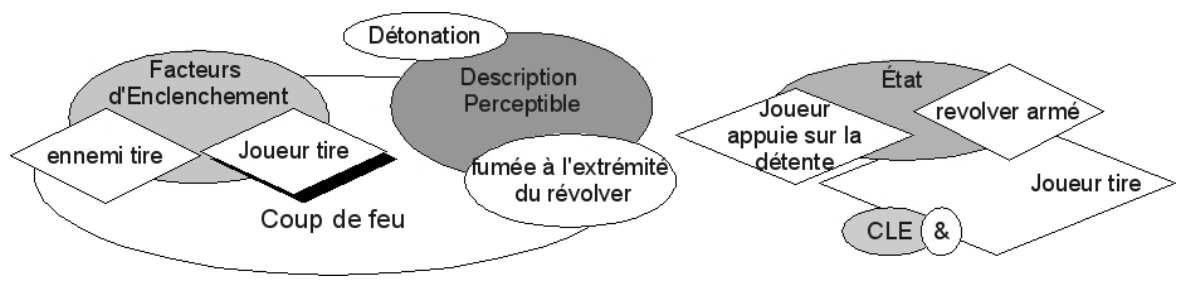

Figure 24. Exemple d'entités validées simultanément

L'auteur peut définir la position spatio-temporelle des entités à l'aide des facteurs d'enclenchement des molécules diégétiques. Ainsi, lorsque deux molécules ont des 
facteurs d'enclenchement identiques, les entités qu'elles représentent sont validées simultanément. De même - dans l'exemple de la figure 24 - "détonation» et « fumée à l'extrémité du revolver » sont des entités enclenchées en même temps, soit lorsque «l'ennemi tire», soit lorsque le «joueur tire» (ce dernier cas de figure se produit si le «joueur appuie sur la détente » et que le « revolver est armé »).

Le scénario, s'il induit une forme de scénation à travers ses facteurs d'enclenchement, et les conjonctions logiques liées aux événements et aux conjonctures, peut aussi préciser la position spatiale de certaines entités. Ces indications sont incluses dans les descriptions perceptibles ou peuvent être définies par l'intermédiaire d'événements spécifiques. La définition des conjonctures - facteurs d'enclenchement - et des événements induisent une scénation variable, à l'intérieur même des entités du document. Ceci permet la description d'entités autonomes dont l'évolution entraine la modification de la scénation. Ce ne sont plus les actions de l'utilisateur qui, seules, modifient l'évolution du document mais la conjonction des actions de l'utilisateur et de celles produites par les autres entités du document. De plus certains événements, dans le cadre de documents à données génératives, peuvent modifier les données. Ces documents sont alors des documents dynamiques.

\section{Autonomie du document}

Les entités d'un document à données génératives sont «vivantes » dans le sens où elles peuvent évoluer en fonction du déroulement du document. Elles sont dynamiques.

\subsection{Documents et entités dynamiques}

Une entité est dynamique si ses caractéristiques sont susceptibles de varier ou de modifier l'état d'autres entités au cours de la lecture du document. C'est le cas notamment en réalité virtuelle où la visualisation du décor est élaborée en temps réel, à partir d'une base de données réduite, ou aussi lorsque certains états (comme le diagnostic de la présence d'un lecteur malvoyant) permettent de modifier l'affichage des entités visuelles ou auditives.

Les molécules diégétiques sont la représentation d'entités autonomes. Par analogie à l'intelligence artificielle, elles énoncent des bases de faits déclaratifs (molécules et atomes diégétiques définis à travers les descriptions sémantiques et perceptibles) et procéduraux (molécules et atomes d'actions contenus dans les molécules diégétiques). Dès lors, les molécules permettent une description dynamique des entités. Ces dernières peuvent s'enclencher, s'afficher, en fonction de la conjoncture générale du document. C'est le rôle des facteurs d'enclenchement. En résumé, les entités contiennent leurs propres modes opératoires (facteurs d'enclenchement et actions). 
L'évolution d'une entité du document résulte de la façon dont elle a été définie et/ou d'événements extérieurs, représentation des actions potentielles de ces entités, de leurs états et de leurs évolutions. Par exemple, l'espace du jeu de Tetris évolue constamment au fur et à mesure du déroulement de la partie. En effet, les formes qui arrivent modifient la zone de jeu, c'est-à-dire l'espace potentiellement accessible à de nouvelles formes.

Il est bon de noter que certaines entités peuvent évoluer sans pour autant être affichées. Ainsi, une entité peut représenter le parcours du lecteur à travers un document ; or, même si elle n'est pas visualisée, l'entité « parcours » évolue dès que le lecteur circule dans le document.

Par ailleurs, la représentation d'entités dynamiques, alliée à la définition d'un sens hypothétique produit par leur affichage, permet d'envisager leur enclenchement par proximité sémantique (le créateur définit ce sens à partir d'hypothèses qui relèvent de choix, éventuellement artistiques, et qui s'avèrent souvent arbitraires). Le document pourra ainsi suivre une évolution cohérente vis-à-vis des objectifs du créateur en validant successivement des éléments proches sémantiquement ou, à l'inverse très éloignés : certaines situations peuvent en effet entraîner des ruptures sémantiques fortes. Une telle conception conduit à la réalisation de documents interactifs qui ne reposent plus exclusivement sur un schéma de mise en situation directement lié aux actions et aux réactions matérielles du lecteur. Dans un tel contexte il ne s'agit plus de concevoir des documents avec des interactions mais une réelle interactivité.

\subsection{Modèle du lecteur (ou modèle de l'interacteur)}

La plupart des documents actuels ont un niveau d'interactivité assez faible. Ceci se matérialise par des commandes d'actions-réactions directes entre le document et son lecteur (l'incidence sur l'affichage de l'action du lecteur est instantanée). Ainsi un clic-souris, un « drag and drop », un « rollover » entraîne une réaction immédiate du système documentaire.

Or, il est préférable - dans un souci de respect des objectifs de l'auteur, tant au plan artistique qu'au plan de la communication - de prendre en considération le comportement du lecteur pour faire évoluer le document multimédia. Certes, l'usager, son rapport au document, sa perception du document sont pris en compte dans la majorité des conceptions de documents multimédias, notamment à travers la conception des interfaces (Pignier et Drouillat, 2008). Les interfaces s'appuient de plus en plus sur des métaphores visant surtout à faciliter l'ergonomie de la navigation non plus d'un strict point de vue des spécialistes de la «communication homme-machine », mais en y intégrant les fonctions d'estime nécessaires à l'adhésion du lecteur au document. 
Toutefois, un auteur, qui plus est s'il est artiste, ira au-delà de cet aspect fonctionnel des relations entre le lecteur et le document. Il aura envie d'innover et d'avoir les moyens d'expression nécessaires à la réalisation du document qu'il a rêvé, du « jeu » de situation qu'il veut créer entre l'interacteur et le document qu'il a mis en place.

L'autonomie des entités permet la représentation d'un modèle de l'interacteur. Il ne s'agit en aucun cas ici de réifier le lecteur mais d'en proposer un «avatar $»^{16}$. Il s'agit de circonscrire un état psychologique du lecteur en fonction de ses réactions. Ceci se fera souvent de façon totalement arbitraire, sans tenir compte d'un quelconque modèle psychologique scientifiquement établi, comme c'est le cas dans l'exemple de la figure 25. Le modèle ne sert qu'à établir une stratégie d'évolution. Un romancier, un scénariste ou un réalisateur de cinéma vont écrire en fonction des réactions potentielles d'un lecteur, d'un spectateur imaginaire. Dans le même ordre d'idée, un modèle de l'interacteur va permettre à l'auteur d'établir une stratégie d'interaction entre les entités du document et l'interacteur.

Aujourd'hui, il est difficilement envisageable de déterminer directement et en temps réel, les états cognitifs et émotionnels d'un utilisateur. Cela implique, si l'on veut faire évoluer le système en fonction du lecteur, de passer par des modèles de représentation de celui-ci. En fait, un modèle du lecteur est une entité qui cherche à caractériser le lecteur à partir de faits objectifs et mesurables par le document et son environnement technique. Le caractère déduit peut ainsi influencer le déroulement $\mathrm{du}$ document. Éventuellement, l'auteur pourra déléguer à des spécialistes de l'intelligence artificielle la conception d'un système spécifique de diagnostic d'un état psychologique, cognitif de l'interacteur lors de la réalisation du document.

Néanmoins, et particulièrement dans un contexte de création artistique, un concepteur peut vouloir faire évoluer le document en fonction du comportement du lecteur face au document, sans se préoccuper d'un quelconque modèle scientifique. Il peut s'aider pour cela de la représentation d'un modèle du lecteur avec des molécules diégétiques qu'il aura construites. Le modèle du lecteur qui suit s'inscrit prioritairement dans ce cadre. De plus, la prévision d'évolution d'un document en fonction d'un état imaginé du lecteur, peut générer des solutions simples de diagnostic d'état de l'interacteur. Ainsi, un auteur peut décider que le document considérera un lecteur nerveux si celui-ci clique à tort et à travers. À l'inverse si le lecteur n'a aucune action le document le considérera comme passif. Développé, ce modèle de l'interacteur peut permettre d'élaborer une stratégie ou un mode d'évolution d'un document.

Ce type de modèle, à partir de règle simple, a permis à des groupes d'étudiants de scénariser des documents établissant des stratégies d'évolution face au lecteur ou son avatar au sein du document. Il s'agissait, par exemple, de documents déterminant la présence (ou l'absence) d'un handicap visuel chez le lecteur et afin de lui proposer des entités et une navigation adaptées à son handicap sans interface particulière.

16. Un avatar est une représentation forcément schématique et approximative de l'utilisateur à l'intérieur du système. 


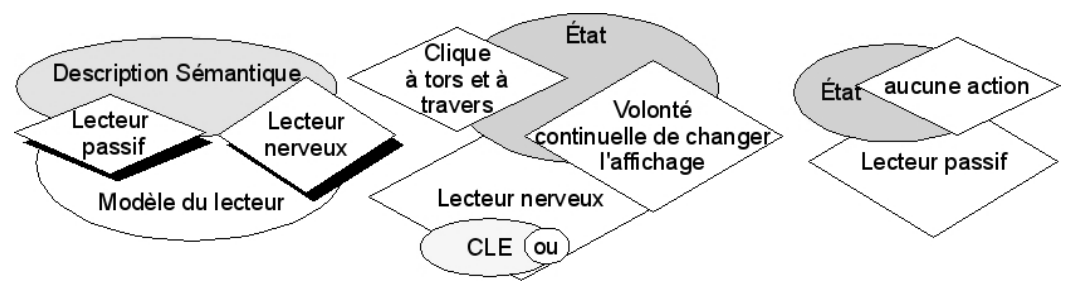

Figure 25. Exemple de modèle de lecteur

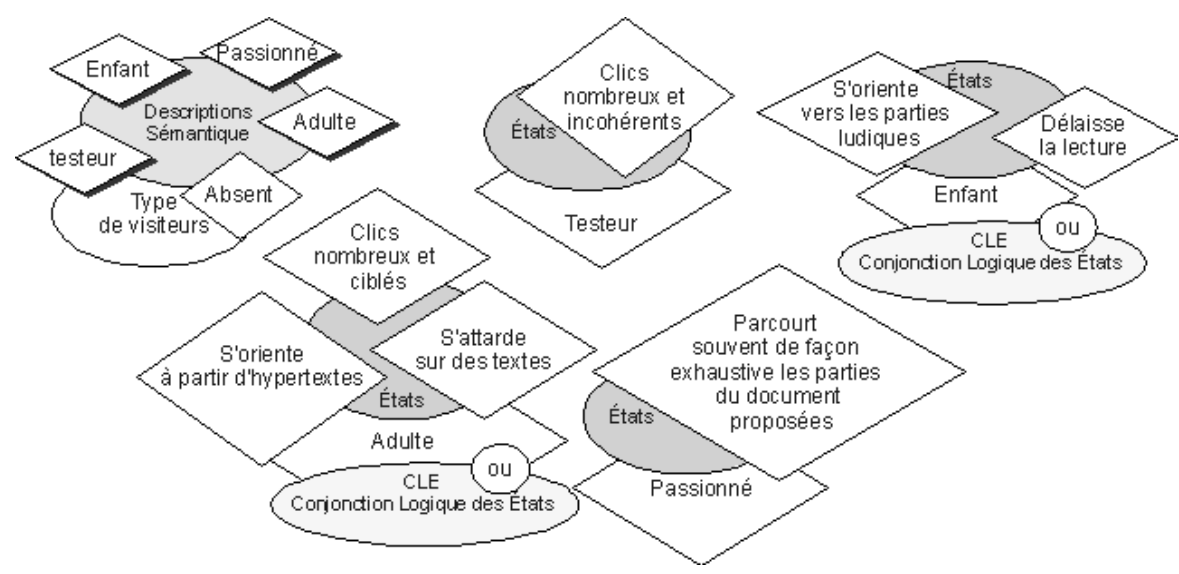

Figure 26. Autre exemple de modèle de lecteur : type de visiteurs

En résumé, le modèle du lecteur n'est pas là pour «fabriquer » un lecteur, mais pour circonscrire - de façon arbitraire, éventuellement avec une vision «d'artiste »un hypothétique état psychologique en fonction de ses réactions. Ce modèle repose sur des faits observables par le document et son environnement technique.

\section{Conclusion}

Le modèle en molécules scénaristiques qui vient d'être présenté permet de représenter un univers imaginaire, avec ses propres lois, sous la forme d'un scénario. Ce dernier définit le document, grâce à la représentation d'entités dynamiques.

Le créateur ne définit plus des liaisons entre données. Il caractérise des entités (objet, lieu, un personnage, réel ou imaginaire, qui participe à l'évolution et/ou à la description de l'environnement). Les entités sont définies par des critères d'états et des caractéristiques d'évolution. Elles sont donc autonomes et peuvent interagir les unes avec les autres. Le lecteur participe à l'évolution du document multimédia à 
travers son avatar : «le modèle du lecteur ». Celui-ci définit un lecteur hypothétique, imaginé par l'auteur, afin de permettre aux entités artificielles du document d'agir en fonction du comportement présumé de ce lecteur imaginaire. En ce sens, l'interacteur constitue une entité de l'environnement. Enfin, la prise en compte d'une description sémantique des entités peut permettre de traiter les entités à partir de significations potentielles. En fait, dans un tel mode de conception, ce ne sont plus les données qui priment mais les informations qu'elles permettent de véhiculer.

Les principes de base exposés dans ce document peuvent paraitre simplistes au premier abord. Toutefois une étude menée sur plusieurs auteurs multimédia montre que l'idée même de documents adaptatifs ou en entités autonomes ne viennent pas à l'esprit des concepteurs. Ces principes sont éventuellement concevables pour des jeux, mais absolument pas pour des documents d'information. Par ailleurs, le mode de perception linéaire des documents empêche souvent les auteurs de "plonger» dans l'écriture d'entités autonomes. Ceci a aussi été mis en évidence progressivement avec des étudiants d'une filière audiovisuelle et multimédia positionnés en tant qu'auteurs, indépendamment de toute réalisation ultérieure effective.

En résumé, l'imagination d'entités qui peuvent être autonomes et la conception de parties de documents, d'unités d'information, en fonction d'utilisateurs différents pose problème. Il y a aujourd'hui un manque de « documents témoins » permettant de corréler le « texte » (dans le sens représentation abstraite) et le document adaptatif avec des entités autonomes effectivement réalisées. Des scénarii «exemples» existent mais le format de cette publication empêche d'en insérer dans ce texte. Pour palier ce problème le lecteur pourra en consulter, publiés sur http://lambouxdurandrech .free.fr/afin de mieux illustrer ces concepts.

Un autre problème, identifié tant par les auteurs «professionnels» que les étudiants (et quel que soit le type de document multimédia réalisé), concerne l'absence d'outil d'écriture adapté. Cet outil - que l'on peut qualifier de «traitement de scénarii multimédias »- est en cours de développement.

\section{Bibliographie}

Allen J.F., "Maintaining knowledge about temporal intervals", Communication of the ACM, 1983, vol. 26, n 11, 1986, p. 832-843.

Allen J.F., Ferguson G. F., “Actions and Events”, Interval Temporal Logic. Journal of Logic and Computation, 1994, vol. 4, n ${ }^{\circ}$ 5, p. 531-579. ftp://ftp.cs.rochester.edu/pub/papers /ai/94.tr521.Actions_and_events_in_interval_temporal_logic.ps.Z

Alvarez J. et al., «Morphologie des jeux vidéo », Hypertextes, hypermédias. Collaborer, échanger, inventer: expériences de réseaux, Paris/Londres, Lavoisier/Hermès Science Publishing, 2007, p. 277-294.

Balpe J.P., « Hypertexte et interactivité », Hypertextes et Hypermédias, Paris, Hermès, vol. 1, $\mathrm{n}^{\circ}$ 1, p. 11-22, 1997. 
Balpe J.P., «Le livre est tout le problème... », Document numérique. Nouvelles écritures. Paris/Londres, Lavoisier/Hermès Science Publishing, vol. 5, n 1-2/2001.

Cartier M., «Un procédurier pour le nouveau Web», 2003. http://www.michelcartier.Com /pdf/Cartier_procedurier.pdf

Coffe J.P., Le marché, CD-ROM, Éditions roule prod. 1995.

Colin M., Cinéma, télévision et cognition, Nancy, Presses Universitaires de Nancy, 1992.

Ferber J., Les systèmes multi-agents. Vers une intelligence collective, Paris, InterEditions, 1995.

Fournier J., Scénarisation multimédia: processus de scénarisation interactive, Québec, Presses de l'Université de Laval, coll. Laboratoire de communautique appliquée, 2003.

Gardies A., Bessalet J., 200 mots-clés de la théorie du cinéma, $2^{\text {nd }}$ éd. Paris, Cerf, coll. $7^{\mathrm{e}}$ art, 1995.

Huart J., Mieux concevoir pour mieux communiquer à l'ère des nouveaux médias : vers des méthodes de conduite de projets et d'évaluation qualité de documents multimédias, Valenciennes, Doct. th. : sciences de l'information et de la communication, Université de Valenciennes, 2000

Jeanneret Y., Souchier E., «Pour une poétique de l'écrit d'écran », Paris, Xoana-Images et sciences sociales, $\mathrm{n}^{\circ}$ 6-7, 1999, p. 97-107.

Leleu-Merviel S., La scénistique : méthodologie pour la conception de documents en media multiples suivant une approche qualité, Saint Denis, Habilitation à diriger des recherches : sciences de l'information et de la communication, Université de Paris VIII, 1996.

Leleu-Merviel S., La conception en communication: méthodologie qualité, Paris, Lavoisier/Hermès Science Publishing, 1997.

Locke J., Essai sur l'entendement humain, 1690. Traduction de P. Coste, édition de 1755 , Éditions É. Naert. Réimpression anastatique de l'édition de 1755. Vrin, « Bibliothèque des Textes Philosophiques.

Meyer B., Conception et programmation orientées objet, Paris, Eyrolles, 2000.

Pignier N., Drouillat B., Penser le Web design: modèles sémiotiques pour les projets multimédias, Paris, L'Harmattan, 2004.

Pignier N., Drouillat B., Le webdesign - Sociale expérience des interfaces web, Paris, Lavoisier/Hermès Science Publishing, 2008.

Rojas E., «Internet, outil de médiation culturelle : vers un modèle de spécification basé sur les figures de médiation", Hypertextes, hypermédias. Collaborer, échanger, inventer : expériences de réseaux, Paris/Londres, Lavoisier/Hermès Science Publishing, 2007, p. 135-151.

Raspiengas J.C., «Bertrand Tavernier: L’ardent sommelier», Télérama n 2469 «spécial Cannes », mai 1997.

Souriau E., L'univers filmique, Paris, Flammarion, 1953. 
80 RSTI - DN - 12/2009. Conception, design des documents numériques

Szilas N., «Le drame interactif: concept, modèle et implémentation », Créations numériques écritures - expériences interactives, Paris/Londres, Lavoisier/Hermès Science Publishing, 2005, p. 209-247.

Viéville N., Écrire pour l'écran - vers un outil d'assistance à l'écriture multimédia, Thèse de Doctorat, Université de Valenciennes et du Hainaut Cambrésis, décembre 2003.

Weissberg J.L., «Qu'est-ce que l'interactivité ? - Eléments pour une réponse », Séminaires $d u$ laboratoire Paragraphe, Paris $8 . \quad$ http://hypermedia.univ-paris8.fr/seminaires/ semaction/ seminaires/txt02-03/fs-03.htm

Zadeh L.A., "Fuzzi sets", Informations and Control, n 8, 1965, p. 338-353. http://wwwbisc.cs.berkeley.edu/Zadeh-1965.pdf 